\title{
Safety Considerations for Operation of Different Classes of UAVs in the NAS
}

\author{
Roland E. Weibel ${ }^{*}$ and R. John Hansman, Jr. ${ }^{\dagger}$ \\ Massachusetts Institute of Technology, Cambridge, MA 02139
}

\begin{abstract}
Recognizing the significant effort underway to integrate UAV operations in the NAS, a preliminary hazard analysis was conducted for two critical hazards of UAV operation. Models were developed to describe UAV ground impact and midair collisions. Under several assumptions, a model of ground impact was used to calculate the UAV system reliability required to meet a target level of safety, for different UAV classes differentiated by mass. The model showed a significantly higher reliability required for high-mass UAVs, and a large variation in reliability required with population density, with a two order of magnitude increase over metropolitan areas. Midair collision risk was estimated in the vicinity of airways using a model of aircraft collisions based on the density of air traffic in those regions. There is a two order of magnitude difference in risk between on-airway and onaltitude operation and operation away from airways and off major flight levels. Therefore, there are potential operating strategies that can reduce the risk of UAV operation, such as procedural separation from high population and high traffic areas. There are also additional mitigation possibilities to further reduce the risk of integrating UAVs in the NAS.
\end{abstract}

\section{Nomenclature}

$\begin{array}{ll}A_{\text {exp }} & =\text { area of exposure } \\ \mathrm{d} & =\text { distance traveled by air traffic within airspace segment } \\ \mathrm{ELS} & =\text { expected level of safety } \\ \mathrm{MTBF} & =\text { mean time Between failures resulting in ground impact } \\ \mathrm{P}_{\text {fat }} \mid \mathrm{coll} & =\text { probability of fatality given a potential collision } \\ \mathrm{P}_{\text {pen }} & =\text { probability of penetration } \\ \mathrm{P}_{\text {mit }} & =\text { probability of mitigation preventing a ground fatality } \\ \rho & =\text { population density } \\ \mathrm{t} & =\text { time } \\ \mathrm{V} & =\text { airspace volume }\end{array}$

\section{Introduction}

$\mathrm{T}$ he success of recent military deployment of Unmanned Aerial Vehicles (UAVs) in Iraq and Afghanistan has both raised public awareness of UAVs and proven their operational viability. This has led to an increasing demand for the ability to operate UAVs for a variety of applications over the United States. However, the current Federal Aviation Regulations did not anticipate the operation of unmanned aircraft in the NAS. Therefore, there are no regulations currently applicable to UAVs. Current UAV operation in the NAS has been limited to flights approved through a lengthy Certificate of Authorization process.

The lack of clear regulations has been a barrier to achieving benefits from potential commercial and civil operations. Recognizing this barrier, a significant effort is currently underway to integrate UAV operations into the National Airspace System (NAS) ${ }^{1}$. One fundamental requirement for operation in the NAS is to preserve the safety of the general public. Therefore, the implications for different classes of UAV operation should be examined based upon the requirement to operate at an equivalent level of safety. The purpose of this paper is to identify potential

\footnotetext{
* Graduate Research Assistant, Department of Aeronautics \& Astronautics, 77 Massachusetts Ave, Room 17-110, AIAA Student Member.

${ }^{\dagger}$ Professor, Department of Aeronautics \& Astronautics, 77 Massachusetts Ave, Room 33-303, AIAA Fellow.
} 
design requirements and approaches for integrating different classes of UAVs into the NAS that meet FAA system safety requirements.

\section{Range of Classifications \& Capabilities}

In considering potential UAV operations in the NAS, it is important to recognize that the label "Unmanned Aerial Vehicle" can be applied to a broad range of vehicle types, configurations, and sizes. This broad spectrum is illustrated in Fig. 1 where several current UAVs are pictured, along with their locations on a logarithmic mass scale.

For the purposes of this analysis, the following classes of UAVs are defined and primarily differentiated by mass: Micro, Mini, Tactical, Medium Altitude, and High Altitude/ UCAV (Unmanned Combat Air Vehicle.). While there is currently not a consensus on classifications for flight

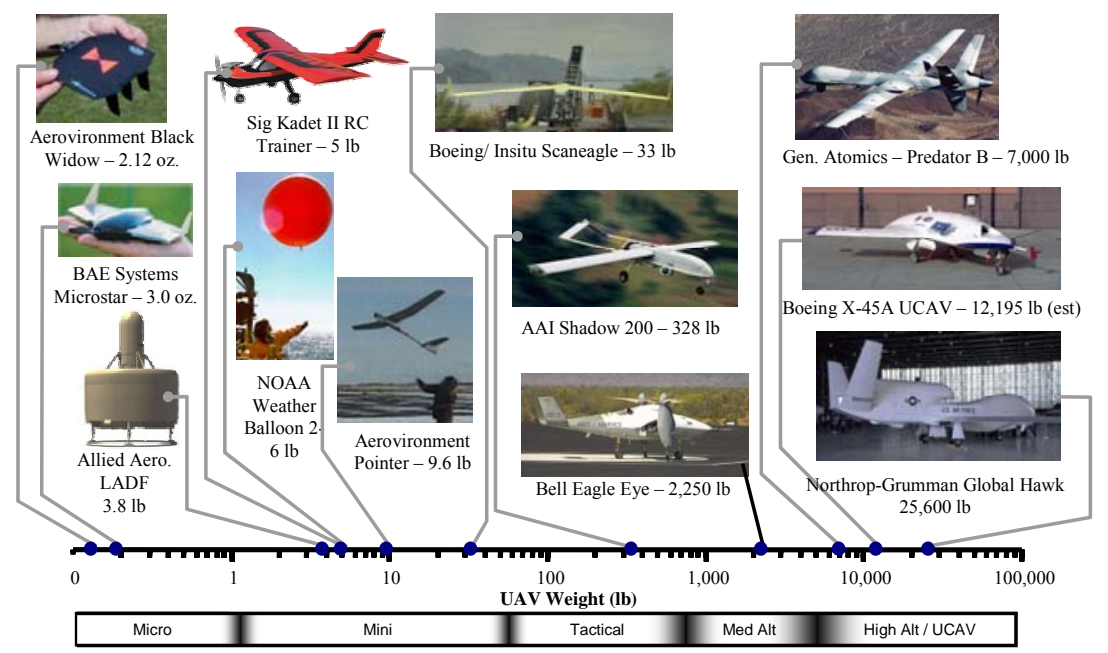

Figure 1. Spectrum of Current UAVs in civil airspace, these definitions are consistent with nomenclature used by both research and military communities. An estimation of the boundaries of each class in terms of UAV mass is also shown in Fig. 1.

The maximum operating altitude of several current UAVs, was assembled from public sources ${ }^{2}$ and is shown in

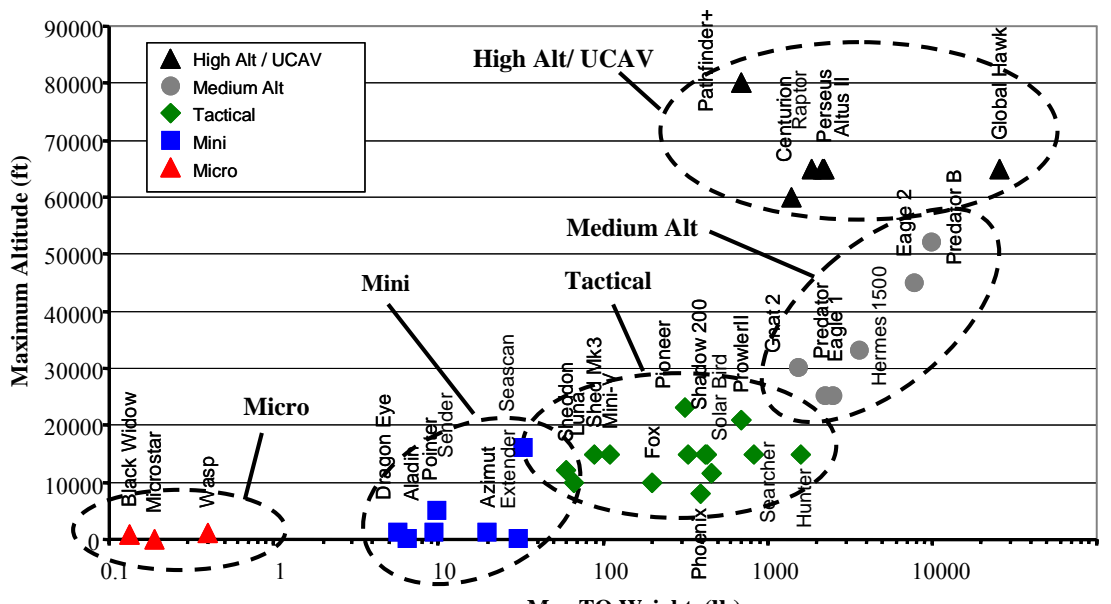

Figure 2 Maximum Altitude of Current UAVs

Fig. 2. There are are several divisions in potential operations between the classes. Micro UAVs are typically limited to short operating ranges, at low altitude, and weigh less than $1 \mathrm{lb}$.. Mini UAVs are operated at intermediate altitudes, and can potentially reach the boundary of controlled airspace at 18,000 ft. Mini UAVs are typically between 1 and $40 \mathrm{lbs}$. The tactical designation is borrowed from military UAV operation, and denotes a UAV that can sometimes be capable of over the horizon operation, and operated at low to intermediate altitudes. Tactical UAVs typically weight between 60 and 1,000 lbs. Medium and high altitude UAVs, as well as UCAV's generally weigh more than $1,000 \mathrm{lbs}$. Medium altitude UAVs are typically operated around the region of Class A airspace, while high alttitude UAVs can potentially be operated above the majority of commercial air traffic.

\section{System Safety Analysis Methodology}

In order to examine the safety implications of several potential classes of UAV operation, a preliminary hazard analysis (PHA) was performed following Federal Aviation Administration (FAA) system safety analysis guidelines ${ }^{3}$. An approach was taken to identify critical hazards associated with operation of a UAV system, develop a model of the expected magnitude of each risk, and determine implications for UAV design and operation. The methodology used is consistent with the system safety literature ${ }^{4}$, and was also informed by FAA advisory circulars for manned aircraft ${ }^{5,6}$. 
To maintain general applicability to a range of UAV systems, a consequence-based approach to the identification of hazards was taken. This approach focused on the most severe classifications of events ${ }^{\ddagger}$. From comparison to manned aircraft reliability requirements, events with the most severe consequences are typically the critical design drivers of the system. Two leading concerns in UAV operation with respect to public safety are ground impact and midair collisions with other manned aircraft. Although other hazards may be present, the critical hazards of ground impact and midair collision will be discussed below.

It is recognized that several factors other than safety will also influence the integration of UAVs into the NAS. Public perception of risks and benefits of UAV operation, as well as the concerns of other stakeholders in the system will also influence FAA safety policy. Nonetheless, safety is expected to remain a fundamental criterion for operations in the NAS.

\section{Ground Impact Hazard}

A model of UAV ground impact was created to investigate the influence of different factors on the expected level of safety in terms of ground fatalities per hour of operation of general UAV systems. The model incorporated total system reliability, UAV size and energy, and population characteristics in the vicinity of operation. The factors are analyzed to determine UAV system reliability requirements to meet a target level of safety.

\section{A. Target Level of Safety}

It is assumed that the ground impact event considered in this analysis will result in a small number of fatalities, and are therefore categorized by FAA criteria ${ }^{3}$ as a hazardous event. The target level of safety of a hazardous event, as specified by FAA requirements for manned aircraft is $10^{-7}$ events per hour of operation ${ }^{3,5,6}$. A review of the NTSB database on air carrier and general aviation accidents showed that this level of safety is consistent with the current level of safety of manned aircraft with respect to ground fatalities, approximately equal to $2 \times 10^{-7}$ ground fatalities per hour of operation over the last ten years ${ }^{7}$. The target level of safety used for the ground analysis was set an order of magnitude beyond that required to manned systems, at $10^{-8}$ fatalities per hour, in realization that UAVs may need to exceed the current level of safety of manned operations.

\section{B. Ground Impact Model}

The ground impact of a UAV was modeled by the basic scenario shown in the event tree of Fig. 3. The ground fatality model presumes that a failure on the vehicle occurs, resulting in an uncontrolled ground impact. The model then considers whether a person is located where the UAV impacts, and if the debris from the crash penetrates the sheltering in which the person is located. Probabilistic analysis of the serial combination of the four events describes the expected level of safety of the system (ELS) in terms of expected fatalities per hour of operation of the system.

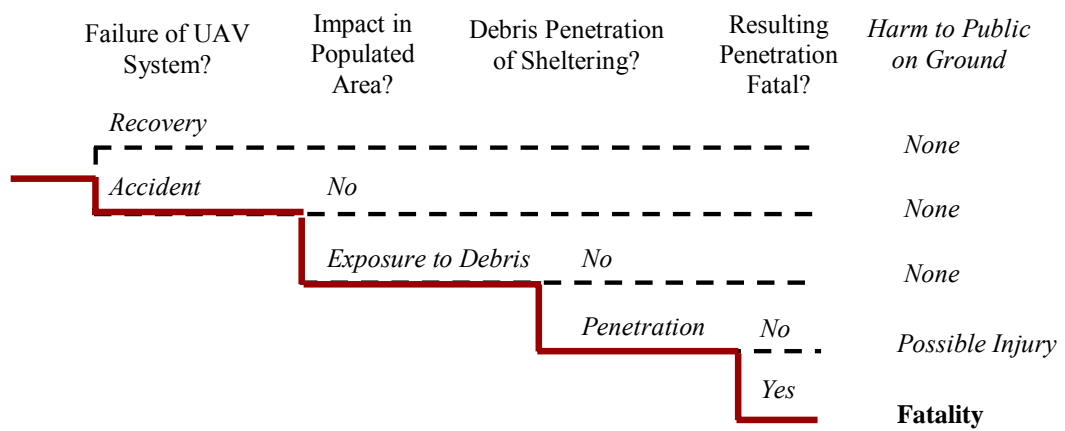

Figure 3. Ground Impact Event Tree
Failures of the UAV system are modeled to occur at an average rate given by a mean time between failures, $M T B F$. The expected number of failures per hour is the inverse of the term. The failures considered are of any general type that could result in ground impact, including failures of any component of the UAV system or human error.

Ground impact of the UAV "exposes" the general public to harm, but does not necessarily directly result in a fatality. The exposure effect was modeled using a debris-footprint based exposure model, developed and used for assessing the risk to the general public from space launch activities ${ }^{8,9}$. By this formulation, the UAV accident exposes a certain area of the ground to harm, denoted, $A_{\text {exp }}$. Then, the expected number of people exposed to harm is the product of the area of exposure and the population density of the area, $\rho$, in number of people per square foot.

\footnotetext{
* The two most severe events categories are hazardous and catastrophic. A hazardous event is defined as resulting in "fatal injury to ground personnel and/or general public," while a catastrophic event "Results in multiple fatalities and/or loss of the system." (Ref 3)
} 
The ground impact model also incorporates population sheltering effects, consistent with previous applications. This aspect of the model recognizes that not all UAV impacts are fatal. The debris must penetrate sheltering, such as vehicles, houses, or other buildings in which the general public is located. The probability that the debris will penetrate shelter given exposure is model by the penetration factor, $P_{p e n}$.

It should be noted that there are several measures that can mitigate the risk of occurrence of a ground fatality. They can be included at any point of the event tree of Fig. 3. All mitigating events are collected and arranged in a term denoted as the probability of mitigation, $P_{m i t}$. The probability of mitigation reflects the probability that a ground fatality does not occur, due to mitigation measures, even though it has been modeled to occur by the assumed sequence of events. Thus, the expected level of safety of the system is increased by 1 minus $P_{\text {mit }}$. For the baseline case, mitigation is not considered, and the term is set to unity. The resulting formulation for the expected level of safety of a UAV operation with respect to ground impact is given by Eq. (1).

$$
\mathrm{ELS}=\frac{1}{\mathrm{MTBF}} \mathrm{A}_{\exp } \rho \mathrm{P}_{\mathrm{Pen}}\left(1-\mathrm{P}_{\text {mit }}\right)
$$

\section{Model Application}

The ground impact model of Eq. (1) was applied to four UAVs from the HALE, Tactical, Mini, and Micro classifications. A summary of the parameters of the model are shown in Table 1.

The expected level of safety of the system estimated by the ground impact model was assumed to be set by the target level of safety to satisfy system safety requirements. Therefore, the variation of risk is investigated as a function of the mean time between failures to meet the required target level of safety. As previously introduced, the target level of safety used for the analyses was $1 \times 10^{-8}$ fatalities per hour of operation.

The area of exposure of the UAV is estimated as the frontal area of the UAV, and population density data were used from the 2000 U.S. Census ${ }^{10}$ for tract groups. The probability of penetration, $P_{p e n}$, will depend on many factors, including the energy of the vehicle, the amount of energy several structures can withstand, and the distribution of people within those structures. For this general approach, a single factor estimate of the probability of penetration was used. It is realized that the factor will vary from $0 \%$ to $100 \%$ from low to high energy impacts. The preliminary estimates of the probability of penetration for Micro, Mini, Tactical, and Hale UAVs is 5\%,10\%, 25\%, and $90 \%$ respectively.

\section{Results}

As can be seen in Eq. (1), the expected level of safety of a UAV system with respect to ground fatalities is a function of the mean time between failures of the vehicle system. By fixing the expected level of safety at the target level of safety of $10^{-8}$ fatalities/hr of operation, the ground impact model generates the reliability required (in terms of mean time between failures that result in ground impact) to meet the target level of safety. The spatial variation of the required reliability is shown in Fig. 4. The proportion of the U.S. for which the reliability required is within the given range is also tabulated in the legend. It should be noted that some UAV ground impacts may be categorized as catastrophic, and would represent an increase in the reliability required beyond the values shown in the figure.

There are two important trends evident in the results of the ground impact model. First, comparing the four vehicle classes, there is a significant variation in required reliability with the mass of the vehicle. For high-mass, high-velocity UAVs, operation over the majority of the United States would require that the vehicle be designed such that the time between failures is much greater than 10,000 hours. However, Mini and Micro UAVs present much lower risk to the general public, and could be operated over the majority of the United States with less than 100 hours required between failures. Tactical UAVs represent an intermediate level of risk.

The second trend evident in the figures is the two order of magnitude increase in reliability required to overfly populated areas. This is most evident in the reliability requirements for tactical and mini UAVs, where one can easily identify the increased population density around major cities such as New York, Chicago, Dallas, Denver, and 
Los Angeles. Recognizing this trend, one possible low-risk integration strategy is to segregate UAV operations from high population areas.

It should be noted that this preliminary analysis did not include possible mitigation measures, such as flight termination systems, emergency parachute recovery systems, or other measures that would lessen the severity of ground impact. Inclusion of such capabilities in aircraft could also be used to achieve an acceptable level of safety.

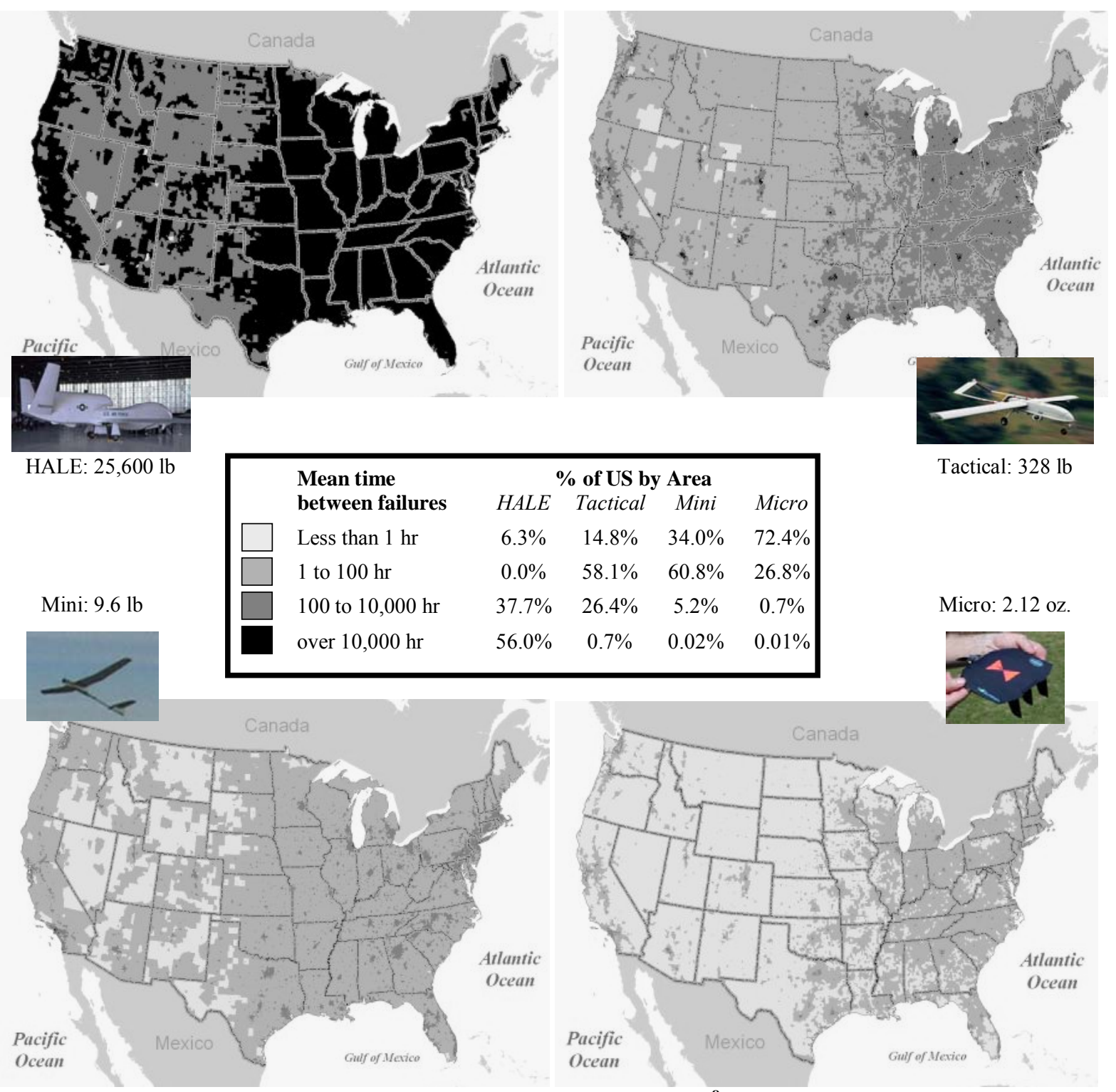

Figure 4. Reliability Required to Meet a Target Level of Safety of $\mathbf{1 0}^{-8}$ fatalities / hr 


\section{Midair Collision Hazard}

A model of midair collisions between UAVs and other aircraft was developed. The model incorporates air traffic density data from an FAA surveillance source to determine the expected number of collisions per hour of UAV flight. The target level of safety for the midair collision hazard from FAA safety guidelines for manned aircraft, is $10^{-9}$ collisions per hour ${ }^{3}$. The expected level of safety was investigated in several regions of the NAS, first averaged from sea level to $50,000 \mathrm{ft}$, and then around jet routes and victor airways. The analysis provides insight into the variation of collision risk with respect to structure of the NAS and the possibility of low-risk operating strategies and requirements for mitigation.

\section{A. Midair Collision Risk Model}

The potential for midair collision between a UAV and several other aircraft was modeled based on a gas model of aircraft collisions ${ }^{11}$. In this model, the UAV is equally likely to be located anywhere in the volume of airspace under investigation. Additionally, its velocity is assumed to be small compared to the threatened aircraft. When threatened aircraft fly within the volume of airspace, they extrude potential collision volumes. The expected level of safety in terms of potential collisions per hour of UAV operation is then the ratio of volume extruded by threatened aircraft per hour to the volume of airspace.

The midair collision model is shown in Fig. 5. Each aircraft flies a distance, $d_{i}$ through the airspace segment under consideration. Each threatened aircraft also has an area of exposure, $A_{\text {exp }}$, representing the contact area that is vulnerable to a collision. For the preliminary analysis the area of exposure was estimated as the frontal area of a 757, approximately 560

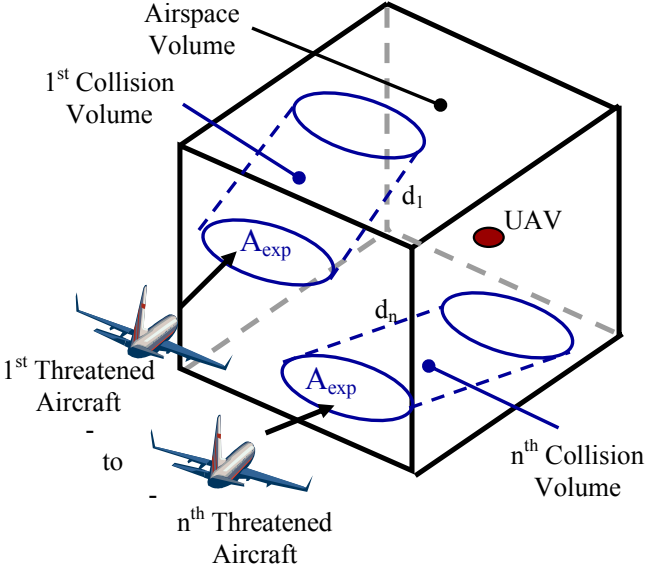

Figure 5. Midair Collision Model $\mathrm{ft}^{2}$. The area of exposure also does not vary significantly with UAV classification, assuming the UAV area is small compared to the threatened aircraft.

The area of exposure, extruded over the distance flown represents a potential collision volume. The total collision volume for threatened aircraft is the area of exposure times the sum of the distances flown by aircraft in the airspace under consideration. Because an expected collision occurs if the exposure volume overlaps with the UAV, the expected number of collisions is equal to the ratio of total collision volume to the volume of airspace.

To generate a sufficient sample of the average behavior of air traffic, the total distance flown is calculated over a given period of time, $t$. The expected collision rate is equal to the expected number of collisions divided by time. However, though a potential collision may occur, there is the possibility that it may not result in fatality, either by direct avoidance of the collision, or by mitigating the magnitude of the collision. Recognizing this, an additional mitigation term is included in the model, $P_{\text {fat coll }}$, which is the conditional probability that the collision is fatal given that there was an expected potential collision. Combining all terms gives the expected level of safety in terms of fatal accidents per hour, shown in Eq. (2). For the baseline analysis, mitigation was not included, therefore the final probability term is assumed to equal unity.

$$
\mathrm{ELS}=\frac{\mathrm{A}_{\text {exp }} \mathrm{d}}{\mathrm{Vt}} \mathrm{P}_{\text {fatcoll }}
$$

\section{B. Data Source}

In order to investigate air traffic patterns in the NAS, data were obtained from the FAA Enhanced Traffic Management System (ETMS) on all surveilled flights over the U.S. for one day in January 2004. The data are organized as position and altitude surveillance gathered from both primary and secondary radar returns and represent all aircraft visible to air traffic control. Where radar coverage is not available, especially at low altitude where obscured from terrain, flight trajectories are not included in the database or are incomplete. Some vehicles which are not tracked by the system, such as ultralights and some general aviation aircraft are not included. Therefore, the dataset represents an under-sampling of traffic density in the NAS. Additionally, as traffic density is averaged over 24 hours in the analysis, the results may also represent an underestimation of the expected level of safety per hour. 


\section{Average Midair Collision Risk over the United States}

To develop a preliminary estimate of midair collision risk, the variation of expected level of safety spatially over the United States was investigated, assuming that the UAV was equally likely to be located from sea level to $50,000 \mathrm{ft}$, neglecting effects of elevation. The model of midair collisions given by Eq. (1) was applied to all air traffic from sea level to $50,000 \mathrm{ft}$ over the United States. The resulting expected level of safety over several regions of the country is shown in Fig. 6.

The results highlight several spatial trends in traffic density, and proportionally, expected level of safety. First, the majority of the collision risk is concentrated over metropolitan areas with major airports by approximately an order of magnitude. Second, the structure of air traffic in the NAS is clear, with large collision risk along several well-traveled routes. The expected level of safety calculated using this method does not adequately capture the expected level of safety in low density regions. The structure of operations on flight levels and along airways is likely to create local regions of increased density in dimensions not analyzed by this method.
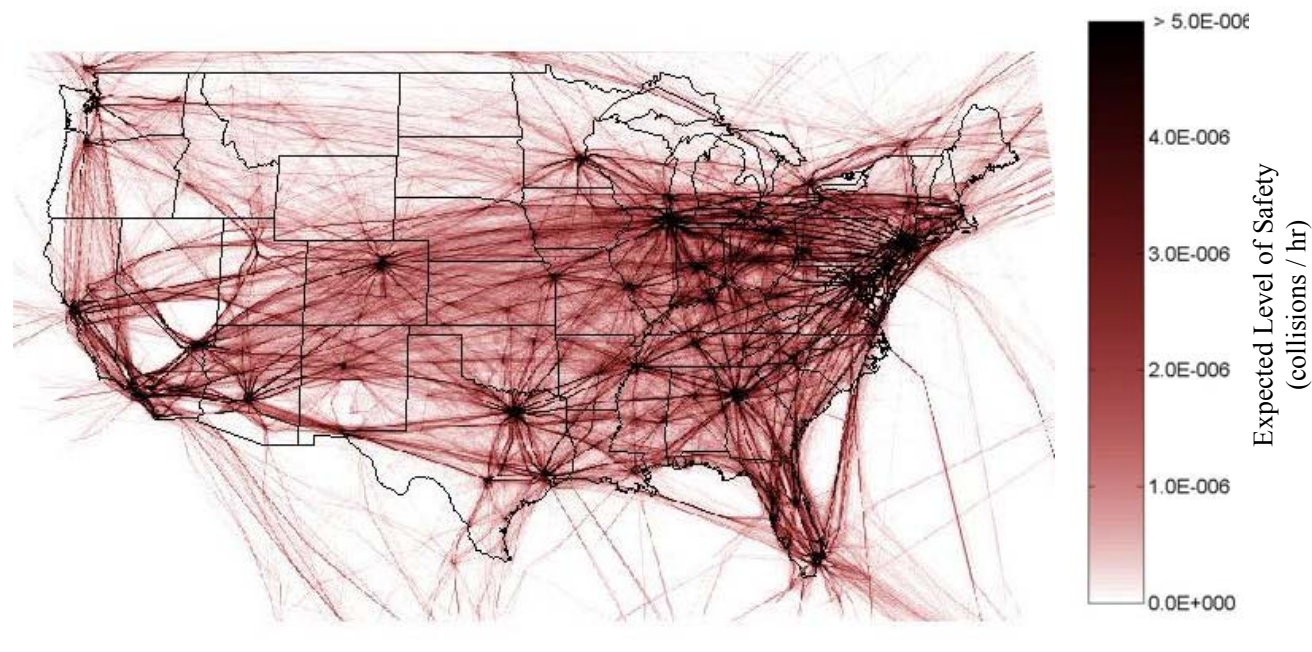

Figure 6. Average Expected Level of Safety from 0 to $50,000 \mathrm{ft}$

\section{Airway Structure}

The preliminary analysis of the previous section identified that the baseline expected level of safety of UAV operation in the presence of other air traffic would not meet the FAA target level of safety. The previous analysis rested on the assumption that the UAV location in the airspace was random. However, the structure of the NAS will likely create locally high densities of aircraft along airways and on flight levels, and a lower than average density in other regions. Therefore, it might be possible to significantly reduce the ambient risk of UAV operation by requiring the UAV to be operated away from airways and major flight levels.

To determine the amount of variation in expected level of safety of UAV operations with respect to airspace structure, traffic density was investigated in the vicinity of jet routes and victor airways in the United States. Potential areas of operation with respect to airway structure are shown in Fig. 7. The four regions of operation for each type of airway form a matrix, described by either being on or off a major flight level (in the altitude dimension), or on or off the airway (in the cross-track dimension).

The width of a victor airway is defined by the FAA as 4 $\mathrm{nm}$ from centerline to boundary ${ }^{12}$. Jet routes do not have a defined width, but $4 \mathrm{~nm}$ was used to remain consistent between analyses. Traffic density was analyzed within $20 \mathrm{~nm}$ on either side of the airway centerline, and results will be shown within 15 miles of the centerline to remove boundary effects. Expected level of safety beyond $20 \mathrm{~nm}$ from the

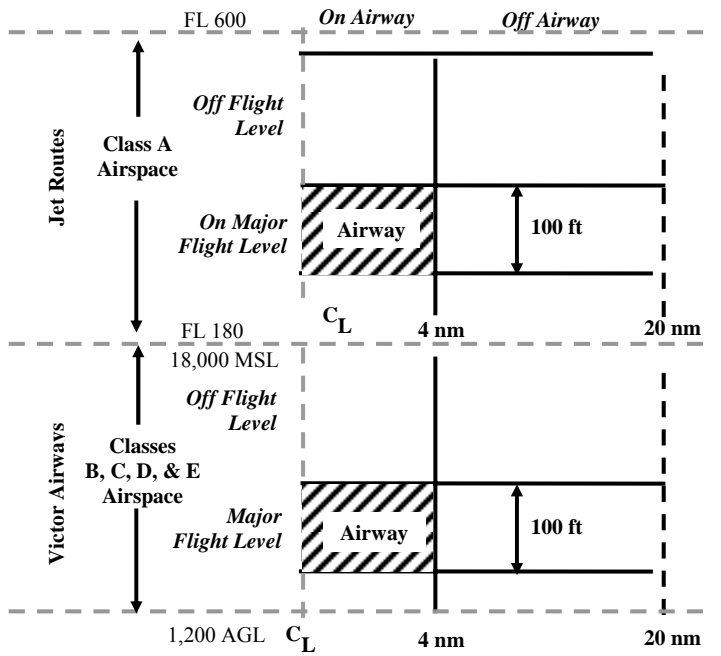

Figure 7. Conceptual Areas of Operation in the Vicinity of Airways 
airway centerline was not investigated.

Traffic density, and therefore collision risk, is expected to be highest on major flight levels and within the airway boundaries, reflecting the operation of the majority of air traffic along airways in the NAS. Traffic density is expected to be lowest both off flight levels and away from airway boundaries. Traffic in this region is expected to be in transition between flight levels and airway waypoints (in two dimensions). The remaining two regions, where transition is expected in only one dimension, should have densities somewhere in between the other regions.

To adequately capture behavior within the regions shown in Fig. 7, flights from the ETMS database were transformed from latitude, longitude, and altitude into local airway coordinates, measured by cross-track deviation from the airway centerline, altitude, and length along the airway. A three-dimensional interpolation was then performed to determine the distance traveled along bins measuring $0.25 \mathrm{~nm}$ in width and $100 \mathrm{ft}$ in altitude. Distance flown was aggregated for all aircraft, representing a measure of the flight density in each bin. The midair collision model was then applied and the expected level of safety was averaged along the length of all airways jet routes and a subset of victor airways in the NAS.

\section{E. Midair Collision Risk in the Vicinity of Jet Routes}

To investigate the variation of collision risk around jet routes, air traffic density was aggregated over all jet routes in the United States, using the method described in the previous section. This included 263 routes, totaling $184,000 \mathrm{~nm}$ in length. The expected level of safety in terms of collisions per hour was calculated using the midair collision model of Eq. (2). The variation in expected level of safety in the vicinity of airways is shown in several dimensions in Fig. 8. To maintain general applicability to a wide range of UAV operations, the expected level of safety was averaged over the length of all jet routes, representing the general behavior of traffic around airway structure. Density may vary locally along individual airways due to the behavior of traffic in the area.

Figure 8a shows the contours of expected level of safety with respect to both cross-track deviation and altitude. The different regions of the expected level of safety are evident in the shading of the contour plot. Each dimension is further analyzed in Figs. 8b to 8d. Very high collision risk is washed out in Fig. 8a, denoted by the largest and darkest bin representing a risk above $1 \times 10^{-6}$ collisions / hr. For the baseline case without mitigating action, there are few regions in the vicinity of airways with an expected level of safety below the target level of safety of $10^{-9}$ collisions per hour.

To examine the variation of collision risk with altitude while operating within airway boundaries, the average collision risk within $4 \mathrm{~nm}$ of the airway centerline along flight levels is shown in Figs. $8 \mathrm{~b}$ and $8 \mathrm{c}$. Jet routes show a clear stratification of density, and therefore expected level of safety along major flight levels. The 1,000 ft separation between flight levels from FL 180 to FL 290 and 2,000 ft separation from FL 290 to FL 450 are apparent in both Figs $8 \mathrm{~b}$ and $8 \mathrm{c}$. The highest average collision risk on an airway is at FL 370, and is approximately $4 \times 10^{-5}$ collisions / hr. The lowest collision risk is on the order of $10^{-9}$ above FL 430.

Figures $8 \mathrm{~d}$ and $8 \mathrm{e}$ show the variation of collision risk with distance from the airway for several altitudes on and off major airways respectively. There is a consistent pattern for all altitudes under investigation, with a large increase in the expected level of safety within approximately $2 \mathrm{~nm}$ of the airway centerline, and a constant risk outside of the airway boundaries. The baseline expected level of safety is on the order of $2 \times 10^{-7}$ collisions / hr, off airways and off major flight levels.

There is a two order of magnitude difference between regions on major flight levels and airways, and off flight levels, away from the airway. The overall collision risk estimates still do not meet the target level of safety. However, it should be noted that these numbers do not reflect any avoidance maneuvers undertaken by the aircraft. To meet the FAA standard target level of safety, those measures would have to add two orders of magnitude to the target level of safety beyond the baseline level. The area above FL 450, although not shown in the figure, has a collision risk on the order of $10^{-9}$ collisions / hr over all regions. Thus, an initial strategy of operating UAVs above FL 450 can have great advantages, as long as control strategies for ascent and descent through high-density regions are undertaken to reduce risk. 

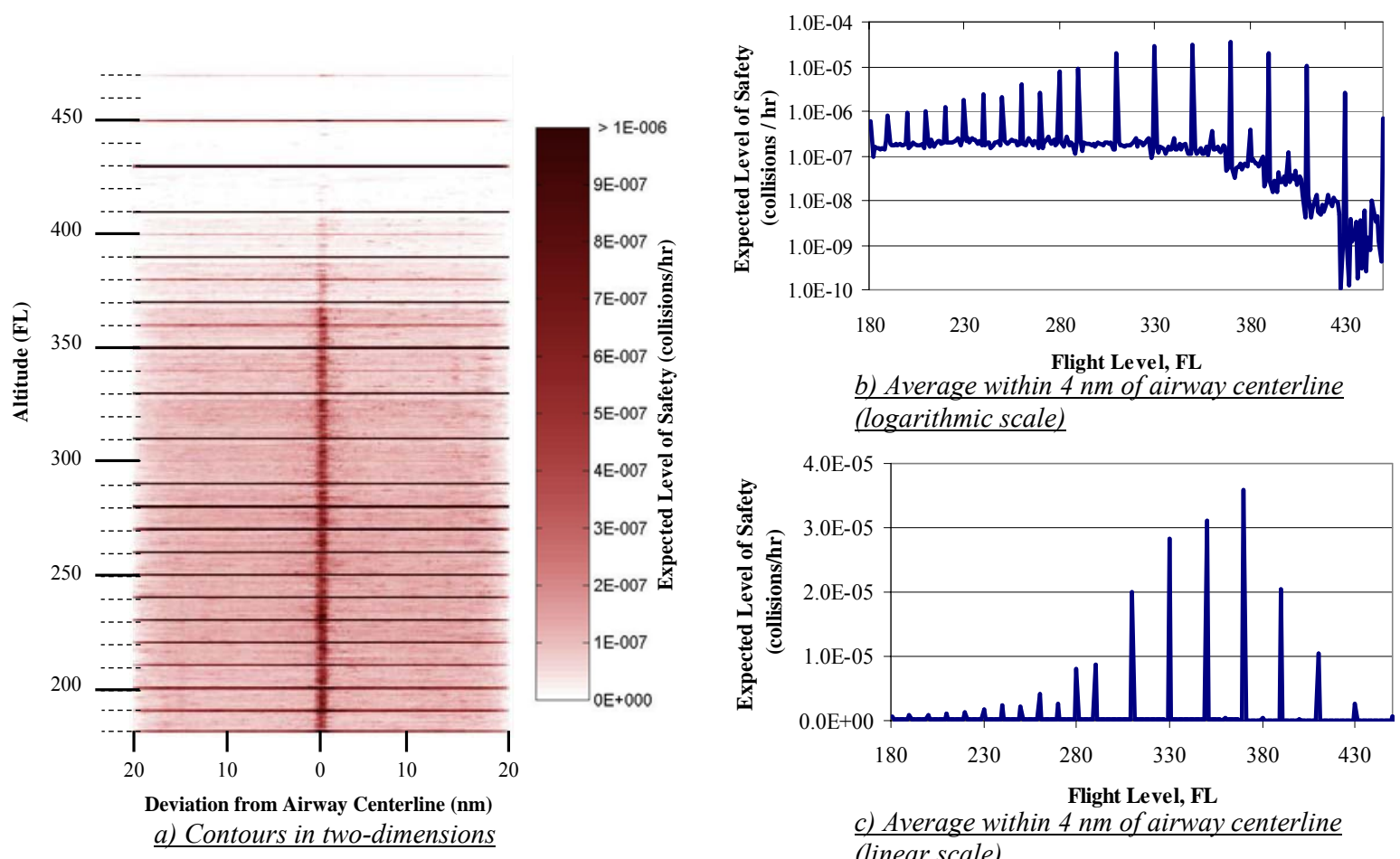

c) Average within $4 \mathrm{~nm}$ of airway centerline (linear scale)
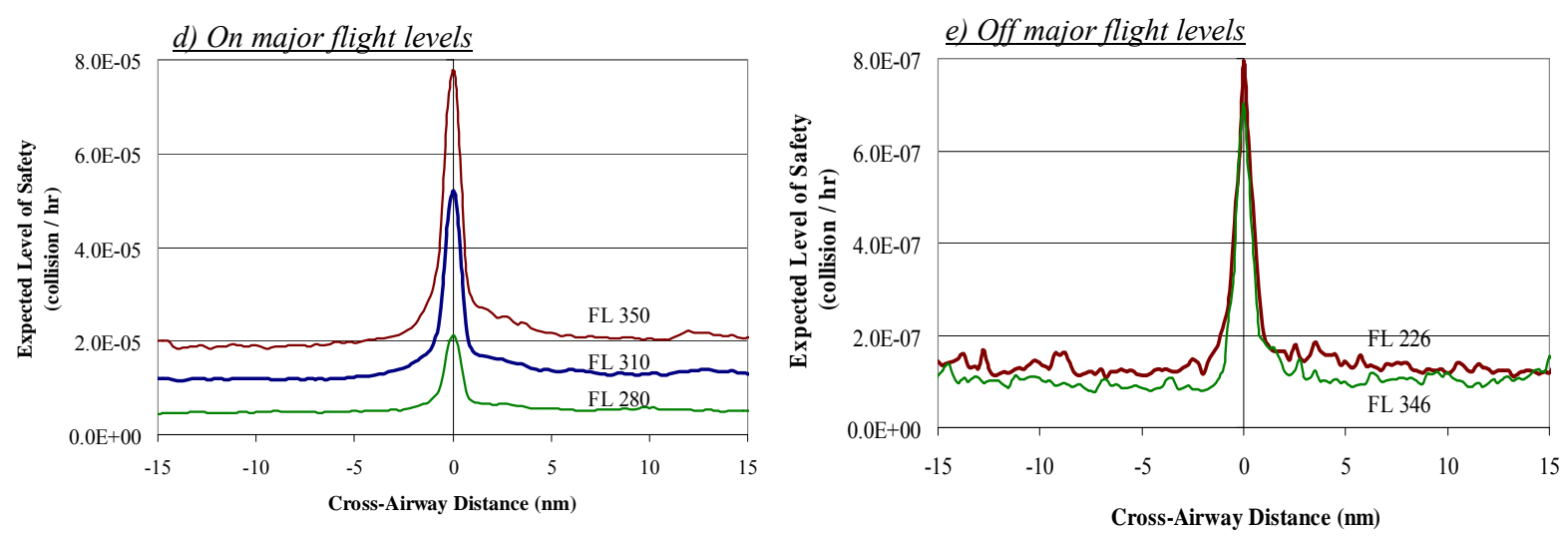

Figure 8. Average Expected Level of Safety in the Vicinity of all Jet Routes in the United States

\section{F. Midair Collision Risk in the Vicinity of Victor Airways}

To adequately capture the behavior around victor airways, it was necessary to select an area of the country with sufficient radar coverage at low altitude. It was also desired to find a region with a high density of air traffic. Under these criteria, flight traffic in the vicinity of victor airways within the NE corridor of the United States was analyzed. This included portions of 102 separate airways, totaling 13,000 $\mathrm{nm}$ in length. The average expected level of safety was calculated based on the midair collision model of Eq. (2). The variation in expected level of safety in the vicinity of airways is shown in several dimensions in Fig. 9. To maintain general applicability to a wide range of UAV operations, the expected level of safety was averaged over the length of all victor airways in the NE, representing the general behavior of traffic around airway structure. Density may vary locally along individual airways due to the behavior of traffic in the area, and may be lower in other regions of the country with a lower density of aircraft. The expected level of safety over victor airways exhibits similar stratification to jet routes in both dimensions, as shown in Figs. 9a and 9b.

Figure $9 \mathrm{c}$ shows the variation of collision risk with altitude, averaged within $4 \mathrm{~nm}$ of the airway centerline. Unlike traffic around jet routes, the expected level of safety does not vary significantly with altitude at the lower 
boundaries of airspace, below 5,000 ft. This trend is likely due to the behavior of flights in the vicinity of airports in the region. The majority of traffic at low altitudes is likely to be maneuvering during departure and arrival. Likewise, the high density is likely to extend to ground level, but the traffic dataset is under-sampled in this region due to terrain blockage of radar returns. Above 5,000 ft in altitude, $1,000 \mathrm{ft}$. separation between flight levels is again apparent, and maximum collision is on the order of $7 \times 10^{-6}$ collisions / $\mathrm{hr}$ at $18,000 \mathrm{ft}$.

Figures $9 \mathrm{~d}$ and $9 \mathrm{e}$ show the variation of collision risk with distance from the airway for sample altitudes on and off major airways respectively. Again, the results are dissimilar to jet routes at low altitude, with little variation in risk with distance from airway centerline. However, as altitude increases, there is an increase in collision risk within approximately $2 \mathrm{~nm}$ of the airway centerline. The expected level of safety off airway and off major flight levels is on the order of $3 \times 10^{-7}$ collisions / hr, at both $6,200 \mathrm{ft}$ and 13,300 ft. This is close to the ambient risk off major altitudes and off airway for jet routes.

Similar to the expected level of safety in the vicinity of jet routes, there is a two order of magnitude difference between regions on major flight levels and on airways, and off flight level, away from the airway. The overall collision risk estimates still do not meet the target level of safety. However, it should be noted that the risk estimated for victor airways do not reflect any avoidance maneuvers undertaken by the aircraft. To meet the FAA standard target level of safety, those measures would have to add two orders of magnitude to the target level of safety beyond the initial level of safety. An additional low risk area of operation where behavior was not analyzed may be very close to the ground, in uncontrolled airspace.

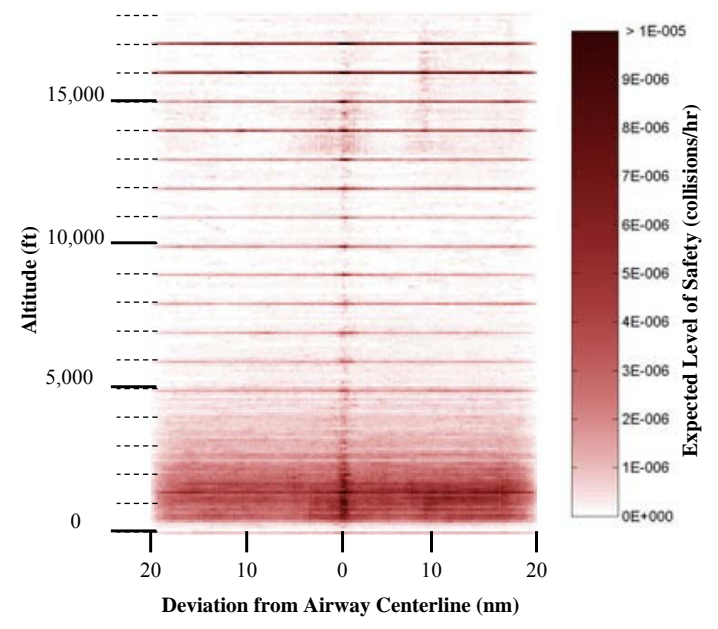

a) On major flight levels

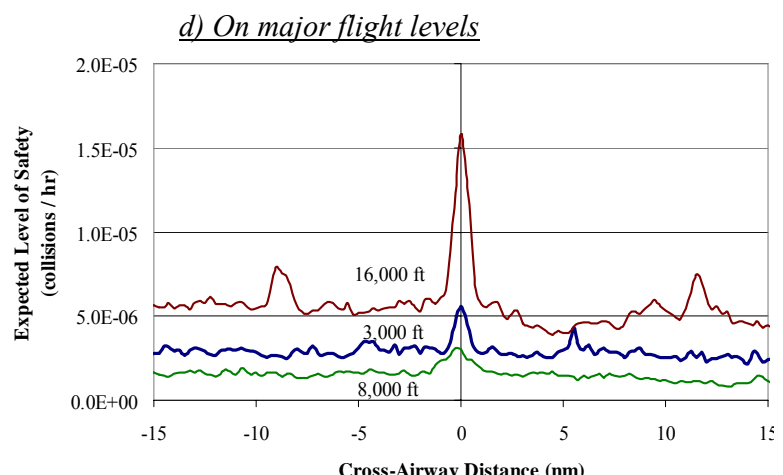

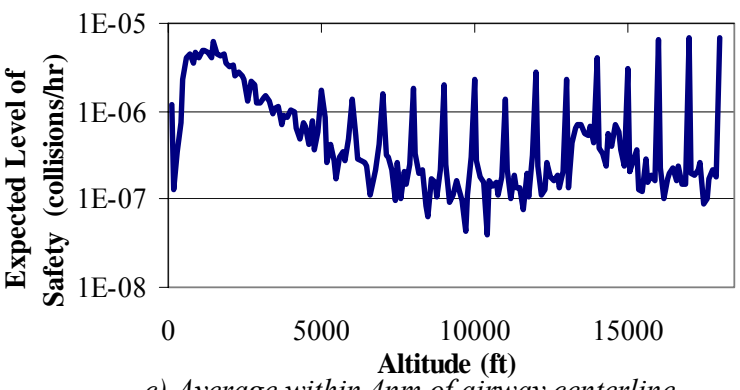

c) Average within $4 \mathrm{~nm}$ of airway centerline (logarithmic scale)

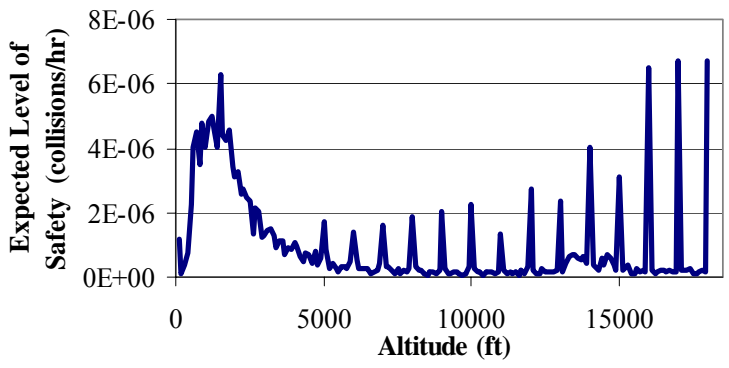

c) Average within $4 \mathrm{~nm}$ of airway centerline (linear scale)

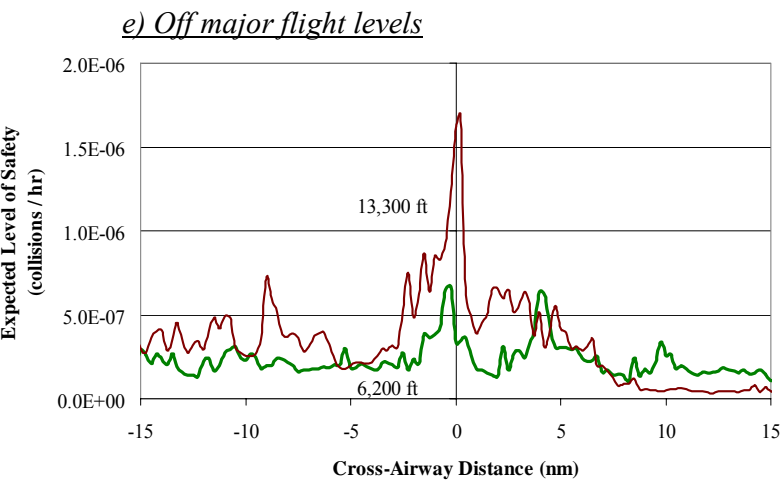

Figure 9 Average Expected Level of Safety in the Vicinity of Victor Airways in the NE Corridor 


\section{Conclusions}

A preliminary hazard analysis was performed for ground impact and midair collisions of UAV systems. Results from models of the event showed significant variation in the expected level. Ground impact risk varied significantly based on population density, and was significantly higher for high-mass and high-velocity UAVs. Midair collision risk varied by two orders of magnitude between flight on airways, on major flight levels and away from airways, off major flight levels.

It was found that it may be possible to operate small UAVs in the NAS, such as those classified as Micro or Mini UAVs, away from only the most densely populous cities. The UAVs could be operated with a mean time between failures of 100 hours, to meet a target level of safety $1 \times 10^{-8}$ fatalities per hour of operation. This reliability is within the capability demonstrated by several current UAV systems ${ }^{13}$. To satisfy midair collision safety requirements, two orders of magnitude of mitigation would be required along with procedural separation of UAVs from high density air routes. Active mitigation measures could include using frangible UAVs that impart less damage an equivalent bird strike, or simple avoidance measures that avoid all aircraft with transponder signals. There is also the opportunity to operate at the boundaries of the NAS, either close to the ground or at very high altitude, where traffic density may be much lower, or the requirements of controlled airspace do not apply.

Significant mitigation would be required to operate higher mass UAVs in the NAS. Under the assumptions of the analysis performed, high altitude UAVs pose a significantly greater risk than other classes, and tactical UAVs represent an intermediate risk level. While the threat posed by higher mass UAVs may be greater, they can also incorporate more sophisticated mitigation measures to prevent midair collisions, such as sense and avoid systems, or active air traffic control separation, an area which is the focus of a great deal of current research. Tactical UAVs inhabit an intermediate region, where a combination of mitigation and procedural separation may be required to meet system safety standards.

Significant future UAV operations are expected to emerge in the NAS. The type and magnitude of mitigation employed to meet system safety standards will vary significantly between classes of UAVs. Integration strategies and mitigation measures applied to large UAVs are not necessarily required or applicable to small UAVs.

\section{Acknowledgments}

This research was supported under the NASA/FAA Joint University Program. The authors wish to thank all of the participants in the Joint University Program at Ohio \& Princeton Universities as well as the students in MIT's International Center for Air Transportation. The following people provided invaluable feedback and insight throughout the investigation process: Jim Kuchar \& Steve Thompson at MIT Lincoln Labs, Mike Paglione \& Phil Potter at the FAA, Frank Frisbie at Northrop Grumman, and Matt DeGarmo at MITRE.

\section{References}

\footnotetext{
${ }^{1}$ Dornheim, M.A., "Flying Well With Others," Aviation Week and Space Technology, Vol. 161, No. 5, 2004, pp. 54-56.

${ }^{2}$ Munsen, K (ed.), Jane's Unmanned Aerial Vehicles and Targets, Various Vols, Jane's Information Group, London.

${ }^{3}$ Federal Aviation Administration, System Safety Handbook: Practices and Guidelines for Conducting System Safety Engineering and Management, December, 2000.

${ }^{3}$ Leveson, N., Safeware: System Safety and Computers, $1^{\text {st }}$ ed., Addison-Wesley, Boston, 1995, Chaps. 13-14.

${ }^{5}$ Federal Aviation Administration, "System Design \& Analysis," AC25.1309-1A, June 1988.

${ }^{6}$ Federal Aviation Administration, "Equipment, Systems, and Installation in Part 23 Airplanes," AC23-1309-1C, March 1999.

${ }^{7}$ National Transportation Safety Board. "NTSB Reports Increase in Aviation Accidents in 2003," Press Release SB-04-09, March 22, 2004.

${ }^{8}$ Baeker, J.B., Collins, J.D., and Haber, J.M., “Launch Risk Analysis,” Journal of Spacecraft, Vol. 14, No. 12, December 1977, pp. 733-738.

${ }^{9}$ Lin, M.Y., Larson, E.W., and Collins, J.D. "Determination of Debris Risk to the Public Due to the Columbia Breakup During Reentry," Appendix D.16 Columbia Accident Investigation Board Report, September 2003.

${ }^{10}$ ArcMap Geographic Information Systems Software Package, Ver. 8.3, ESRI Software.

${ }^{11}$ Endoh, S., "Aircraft Collision Models," M.S. Thesis, Department of Aeronautics and Astronautics, Massachusetts Institute of Technology, Cambridge, MA, 1982.

${ }^{12}$ Spence, C.F. (ed.), FAA Aeronautical Information Manual. McGraw-Hill, New York, 2003, Chap. 5.

${ }^{13}$ Office of the Secretary of Defense. "Unmanned Aerial Vehicle Reliability Study," February 2003.
} 